\title{
Exercícios combinados em adultos com deficiência visual
}

http://dx.doi.org/10.11606/1807-5509201700030563

\author{
Rafaella Righes MACHADO* \\ Auriane Aparecida CAMILLO* \\ Cati Reckelberg AZAMBUJA* \\ Kelly Christine Maccarini PANDOLFO* \\ Sara Teresinha CORAZZA* \\ Daniela Lopes dos SANTOS* \\ *Universidade Federal \\ de Santa Maria, Santa \\ Maria, RS, Brasil.
}

\section{Resumo}

0 objetivo deste estudo foi avaliar o efeito de um programa de exercícios físicos combinados no tempo de atividade física e qualidade de vida de adultos com deficiência visual (DV). Participaram nove adultos com DV com média de idade de 44,9 911,3 anos. Foi realizado um programa de treinamento com exercícios aeróbicos e de treinamento com pesos combinados em uma única sessão, duas vezes na semana, por um período de 12 semanas, em um Espaço de Atividades Físicas. 0 tempo de atividade física (TAF) foi avaliado por meio do IPAQ, versão curta e a qualidade de vida (OV) pelo WHOQOL - BREF abreviado na versão em português. Também foi aplicada uma Ficha Anamnética para caracterizar os participantes. As avaliações foram realizadas anteriormente o início do treinamento e ao término do mesmo. Foi utilizado o teste Shapiro-Wilk para verificar a normalidade dos resultados e o teste Wilcoxon para avaliar a diferença entre as médias obtidas antes e após o treinamento. Foram observadas melhoras estatisticamente significativas no TAF e nos domínios físico e psicológico da QV. Conclui-se que a prática de exercícios físicos combinados promove melhoras nos niveis de QV e TAF, possibilitando um estilo de vida mais ativo e maior independência para pessoas com DV.

PalavRas-chave: Exercício Aeróbico; Exercício Resistido Funcional; Tempo de Atividade Física; Qualidade de Vida; Pessoas com Deficiência.

\section{Introdução}

Conforme o censo do $\mathrm{IBGE}^{1}$, ao que se refere a população brasileira com deficiência, apenas $10 \%$ destas pessoas estão engajadas na prática de exercícios físicos. A deficiência visual (DV), segundo Munster e ALMEIRA " "é caracterizada pela perda parcial ou total da capacidade visual, em ambos os olhos, levando o indivíduo a uma limitação em seu desempenho habitual" (p. 29). Segundo a OrganizaÇÃo Mundial DA SAÚDE ${ }^{3}$, a qualidade de vida (QV) pode ser definida como a "percepçáo do indivíduo de sua posição na vida, no contexto da cultura e sistema de valores nos quais ele vive e em relação aos seus objetivos, expectativas, padróes e preocupaçóes" (p. 1).

A prática regular de exercícios físicos é um componente essencial para manter um estilo de vida saudável e uma boa QV, sendo que seus benefícios já estão bem descritos na literatura ${ }^{4-7}$ Entende-se que o exercício físico é um componente da atividade física que se caracteriza por ser estruturado e repetitivo e tem por objetivo melhorar ou manter um ou mais dos componentes da aptidão física ${ }^{8}$. No entanto, estudos demonstram que pessoas com DV não possuem tempo de atividade física (TAF) satisfatórios ${ }^{9}, 10$ e, apesar da DV não afetar negativamente a $\mathrm{QV}^{10,11}$, bons níveis de atividade física estão associados a uma melhor percepção da mesma ${ }^{12-14}$. De uma maneira geral, estudos ${ }^{15,16}$ demonstram que adultos com DV praticantes de atividades esportivas demonstram boa percepção da $\mathrm{QV}$, assim como, idosos participantes de programas de reabilitação $\mathrm{o}^{17,18}$.

Diante disto, outras possibilidades de exercícios físicos destinados à melhoria da $\mathrm{QV}$ e do TAF, podem ser aplicados a esta população. Visando contemplar 
um maior número de capacidades físicas, alguns estudos vêm demonstrando resultados positivos ao desenvolver programas de exercícios físicos combinados destinados a pessoas com outros tipos de deficiência, tais como Síndrome de Down ${ }^{9,19}$, deficiência intelectual ${ }^{20}$ e física ${ }^{21}$.

No entanto, dentre os poucos estudos realizados, evidencia-se uma carência na literatura acerca da realização de programas de exercícios físicos combinados destinados a melhora do TAF e QV

\section{Método}

Foi realizado contato com 31 adultos que possuíam DV, vinculados a uma Associação de Cegos e Deficientes Visuais e em uma Universidade do Sul do Brasil. Destes, 20 atenderam aos seguintes critérios de inclusão: possuíam DV sem outra deficiência associada; tinham idade mínima de 20 anos; não possuíam problemas de saúde que impediam a realização de testes físicos ou a prática de exercícios físicos e residiam no município em que foi realizado o estudo. Apenas 12 pessoas aceitaram participar da pesquisa, sendo que as demais não tiveram interesse, ou não quiseram sair de casa, mesmo acompanhados de uma pessoa vidente ou ainda, não atenderam ao telefone e/ou e-mail, que foram os métodos utilizados para o contato com os possíveis participantes.

Foram excluídos do estudo três sujeitos que não apresentaram $75 \%$ de frequência ao treinamento e de adultos com DV. Ressalta-se a importância de priorizar estudos na fase adulta, uma vez que nesta faixa etária o envolvimento em atividades que demandam maior esforço físico tende a declinar ${ }^{22,23}$. Além disso, de acordo com ŁABUDZKI e TASIEMSKI ${ }^{24}$ as pesquisas realizadas com pessoas com DV geralmente são com crianças ou idosos.

Portanto, o objetivo deste estudo foi avaliar o efeito de um programa de exercícios físicos combinados no TAF e QV de adultos com DV.

que não realizaram todas as avaliações. Desta forma, nove pessoas com DV participaram do estudo, sendo três do sexo feminino e seis do sexo masculino e,

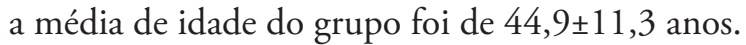

Os voluntários, depois de esclarecidos sobre a proposta do estudo e os procedimentos aos quais seriam submetidos, assinaram o Termo de Consentimento Livre e Esclarecido, que foi lido para os participantes individualmente. O presente estudo foi aprovado pelo Comitê de Ética em Pesquisa com Seres Humanos da Universidade Federal de Santa Maria, estando de acordo com a Resolução 196/96 da Comissão Nacional de Ética em Pesquisa, sob o protocolo no 31104314.2.0000.5346.

A TABELA 1 demonstra as características dos sujeitos que participaram do estudo, tais como, sexo, idade, se possuíam cegueira ou baixa visão, tempo e causa da deficiência e mobilidade no dia a dia.

TABELA 1 - Características dos participantes.

F: feminino; $M$ masculino; BV: baixa visão; CG: cegueira; Adq: adquirida; Desl: deslocamento; NO: nervo óptico.

\begin{tabular}{ccccccc}
\hline Sujeitos & Sexo & Idade & $\begin{array}{c}\text { Tipo da } \\
\text { Deficiência }\end{array}$ & $\begin{array}{c}\text { Tempo da } \\
\text { Deficiência }\end{array}$ & Causa & Mobilidade \\
\hline A & F & 48 & BV & Congênita & Glaucoma & Sozinho \\
\hline B & F & 62 & BV & Adq. há 5 anos & Desl. Retina & Pessoa vidente \\
\hline C & F & 55 & CG & Adq. há 20 anos & Retirada NO & Bengala \\
\hline D & M & 20 & CG & Adq. há 12 anos & Glaucoma & Bengala \\
\hline E & M & 39 & BV & Adq. há 7 anos & Glaucoma & Sozinho \\
\hline F & M & 39 & BV & Adq. há 15 anos & Acidente & Sozinho \\
\hline G & M & 41 & BV & Adq. há 18 anos & Retinose & Pessoa vidente \\
\hline H & M & 51 & CG & Congênita & Desl. Retina & Bengala \\
\hline I & M & 62 & CG & Adq. há 2 anos & Diabetes & Pessoa vidente \\
\hline
\end{tabular}


As sessões de treinamento ocorreram em um Espaço de Atividades Físicas, duas vezes na semana, com duração de 60 minutos cada, por um período de 12 semanas. Cada sessão foi dividida da seguinte maneira: parte inicial, principal e final. Na parte inicial foram realizados 15 minutos de exercícios aeróbicos na esteira elétrica da marca Oxer OXT4100 ou na bicicleta ergométrica da marca Kikos HC3015 ou ainda, no elíptico da marca Athletic Works MTDP408E. A parte principal foi composta por exercícios de treinamento com pesos e materiais de treinamento funcional, organizados em circuito, com duas séries de um minuto de duração e 15 segundos de intervalo entre elas e, 30 a 45 segundos de intervalo para a troca de estações de exercícios, e número máximo de repetiçóes em cada série. Nesta parte foram realizados exercícios para membros superiores, tais como apoios, crucifixos, remadas, rosca direta e alternada, tríceps testa, francesa; para o tronco abdominais e pranchas e, para os membros inferiores, agachamentos, adução e abdução. Os exercícios foram realizados utilizando os seguintes materiais: colchonetes, bolas suíças de $75 \mathrm{~cm}$, bosu, cama elástica, disco de equilíbrio, elástico extensor, funcional suspenso, slide, gymstick, halteres, step, roda de exercícios, medicine ball. Na parte final foram realizados o alongamento dos grupos musculares trabalhados. Os sujeitos foram distribuídos em grupos de no máximo quatro pessoas, de acordo com sua disponibilidade de horário e faixa etária. Cada grupo teve o treinamento sob orientação de duas professoras de Educação Física.

Anteriormente ao início do treinamento foram realizadas duas sessóes de adaptação dos participantes, visando a familiarização com os materiais e/ou espaços físicos e sua locomoção até o local de forma independente, as quais constituíramse da seguinte maneira: reconhecimento do caminho do centro da cidade até o local de realização do programa de treinamento, juntamente com as professoras, reconhecimento do local, familiarização com os materiais e exercícios realizados nas sessōes de treinamento.

A cada semana foram aumentadas a intensidade e a complexidade dos exercícios, por meio dos aumentos da velocidade na esteira, da carga na bicicleta e elíptico, da carga dos exercícios com pesos e da complexidade dos exercícios realizados com o peso do corpo, mantendo o número de séries e o tempo de repetiçóes, conforme a percepção subjetiva de esforço dos participantes, avaliada por meio da Escala de Borg (6-20) ${ }^{25}$.
Diante da limitação da amostra, a Escala de Percepção Subjetiva de Esforço sofreu uma adaptação da seguinte maneira: inicialmente foi separada em três categorias de dificuldade (fácil - de 6 a 10; moderado - de 11 a 15; difícil - 16 a 20). Após, em cada categoria, foi atribuído cinco pontos de dificuldade, onde o número 1 (um) indicava muito difícil e o 5 (cinco) muito fácil, sendo este indicativo de necessidade de progressão de carga. Esta adaptação foi verbalizada a todos os participantes e a intensidade alvo do treinamento foi desenvolvida na zona de classificaçáo moderada. Foram realizadas duas avaliaçóes, sendo a primeira anteriormente ao início do treinamento e a segunda após o término do treinamento, na $12^{\text {a }}$ semana. Para caracterizar o grupo de estudo foi aplicada uma Ficha Anamnética ${ }^{26}$ e, para avaliar o TAF foi utilizado o Questionário Internacional de Atividades Físicas (IPAQ), versão curta ${ }^{27}$. Já a QV foi avaliada por meio do WHOQOL (World Health Organization Quality of Life) - BREF abreviado na versão em português ${ }^{28}$. O módulo WHOQOL-BREF é constituído de 26 perguntas (sendo a pergunta número 1 e 2 sobre a qualidade de vida geral), as respostas seguem uma escala de Likert (de 1 a 5 , quanto maior a pontuação melhor a qualidade de vida). Além dessas duas primeiras questóes, o instrumento tem 24 facetas as quais compóem 4 domínios que são: físico, psicológico, relaçóes sociais e meio ambiente.

O TAF foi quantificado seguindo a protocolo do instrumento com o objetivo de apenas comparar se o exercício físico o influenciou de forma positiva no pós-teste, não sendo utilizada a classificação em categorias. O WHOQOL-BREF foi classificado calculando-se os escores por meio da soma dos valores das facetas e posteriores divisóes pelo número de questóes em cada domínio. Em seguida, obteve-se a média de cada domínio e, logo após, qualificou-se cada um conforme a classificação: necessita melhorar (quando foi 1 até 2,9); regular (3 até 3,9); boa ( 4 até 4,9) e muito boa (5). A Ficha Anamnética ${ }^{26}$ e os questionários foram aplicados na forma de entrevista.

Para análise estatística, verificou-se a não linearidade dos resultados por meio do teste ShapiroWilk, e então, foi utilizado o teste Wilcoxon para avaliar a diferença entre as médias de pré e pós teste, utilizando o Statistical Package for the Social Science (SPSS, Chicago, EUA), versão 21.0, adotando-se um nível de significância de $p<0,05$. 


\section{Resultados}

A TABELA 2 demonstra o TAF e a QV, dividida nos domínios físico, psicológico, relaçóes sociais, meio ambiente, percepção geral da QV e satisfação com a saúde, dos participantes antes e após o treinamento.

Pode-se verificar que os participantes apresentaram melhoras estatisticamente significativas após o treinamento no TAF e nos domínios físico e psicológico da QV. Apesar de não haver diferenças estatisticamente significativas nos demais domínios, pode-se observar uma tendência de melhora nos valores mínimos dos demais domínios, com exceção do domínio meio ambiente. Além disso, após o período de treinamento, os três participantes que dependiam de outra pessoa para locomover-se, passaram a deslocar-se de forma independente. Optou-se por analisar o TAF em minutos/semana e não por categorias, pelo tamanho reduzido da amostra.
TAF: tempo de atividade física; QV: qualidade de vida; Máx.: máximo; Mín.: Mínimo. ${ }^{*} p<0,05$

TABELA 2 - Tempo de Atividade Física e Qualidade de vida dos participantes antes e pós o treinamento.

\begin{tabular}{lccccccc}
\hline \multirow{2}{*}{ Variável } & \multicolumn{9}{c}{ Pré } & \multicolumn{4}{c}{ Pós } & \multirow{2}{*}{$\boldsymbol{p}$} \\
\cline { 2 - 8 } & Máx. & Min. & Média & Máx. & Min. & Média & \\
\hline TAF $(\mathrm{min} / \mathrm{sem})$ & 740 & 115 & $332,2 \pm 218,2$ & 1480 & 280 & $614,4 \pm 355,9$ & $0,008^{*}$ \\
\hline Físico & 4,6 & 2,7 & $3,5 \pm 0,6$ & 4,3 & 3,4 & $3,9 \pm 0,2$ & $0,041^{*}$ \\
\hline Psicológico & 4,2 & 2,5 & $3,5 \pm 0,6$ & 4,2 & 2,7 & $3,7 \pm 0,5$ & $0,030^{*}$ \\
\hline Relaçôes sociais & 5 & 3 & $3,9 \pm 0,7$ & 5 & 4 & $4 \pm 0,3$ & 0,062 \\
\hline Meio ambiente & 4,5 & 2,5 & $3,4 \pm 0,6$ & 4,1 & 2,5 & $3,5 \pm 0,4$ & 0,735 \\
\hline Percepçấo QV & 5 & 3 & $3,8 \pm 0,6$ & 5 & 4 & $4 \pm 0,3$ & 0,157 \\
\hline Satisfação saúde & 5 & 2 & $3,2 \pm 1$ & 4 & 4 & $4 \pm 0$ & 0,068 \\
\hline
\end{tabular}

\section{Discussão}

O treinamento combinado de exercícios físicos aeróbicos e de treinamento com pesos se mostrou eficaz para os participantes da pesquisa ao proporcionar melhoras na QV e no TAF, corroborando com outros estudos realizados, nos quais exercícios combinados em programas de treinamento também demonstraram ser eficazes ao proporcionar ganhos nas atividades funcionais em um adolescente com lesão medular ${ }^{21}$, melhoras no estado nutricional e resistência muscular de adultos com Síndrome de Down ${ }^{9,19,29}$ e melhoras no perfil lipídico, capacidade cardiorrespiratória, força e pressão arterial de adultos com deficiência intelectual ${ }^{20}$.

Os estudos anteriormente citados foram realizados com pessoas com deficiências diferentes da amostra deste estudo, o que pode comprometer a interpretação comparativa dos dados. O ineditismo da pesquisa realizada com DV brasileiros limita bastante a discussão dos achados.

Neste estudo, verificou-se que os participantes aumentaram seus níveis de atividade física, sendo observada diferença estatisticamente significativa entre as médias antes $(322 \pm 218,2 \mathrm{~min} / \mathrm{sem})$ e após
$(614 \pm 355,9 \mathrm{~min} / \mathrm{sem})$ o treinamento, conforme a TABELA 2. Nesse sentido, a prática de exercícios físicos se faz importante, pois sabe-se que baixos níveis de atividade física em adultos com DV estão relacionados a maiores riscos de desenvolvimento de doenças cardíacas, diabetes, hipertensão, depressão, osteoporose, dentre outras ${ }^{30}$.

Além dos benefícios físicos proporcionados pelo treinamento, o fato dos DV participarem ativamente do estudo, melhorando sua mobilidade e consequente autonomia, pode ter contribuído para um aumento do TAF. Sabe-se que muitas vezes a DV priva as pessoas de realizar determinadas atividades do dia a dia de forma autônoma, sendo a locomoção uma delas. Foi possível perceber, no presente estudo, que três participantes que inicialmente dependiam de outras pessoas, passaram a deslocarse de forma independente após o treinamento. Neste sentido, Montarzino et al. ${ }^{30}$ afirmam que a mobilidade tem um papel fundamental na QV de pessoas com DV. No entanto, vale ressaltar, que estar fisicamente e emocionalmente aptas a 
locomover-se sozinhas não se faz suficiente se não há condições de acessibilidade e segurança para tal.

Em se tratando da QV, no presente estudo foram verificadas melhoras estatisticamente significativas nos domínios físico e psicológico após o treinamento, como demonstrado na TABELA 2. No que se refere aos demais domínios, todos os DV poderiam ser classificados como "muito bons" no início do treinamento, ainda assim, foi possível observar tendência de melhora nos valores mínimos, com exceção do domínio meio ambiente.

LIMA et al..$^{15}$ avaliaram a QV de pessoas com DV, na faixa etária de 18 a 55 anos de idade, após três meses de prática esportiva. O grupo experimental composto por 12 atletas, de ambos os sexos, que praticavam futebol e atletismo, apresentaram melhoras estatisticamente significativas nos aspectos sociais, emocionais e físicos, quando comparados ao grupo de sedentários. Assim como no presente estudo, os autores também não verificaram diferenças estatisticamente significativas na percepção de saúde.

Bittencourt e Hoenne ${ }^{11}$ ao avaliar a QV de 16 adolescentes e adultos com DV, frequentadores de um programa de reabilitação de um serviço universitário, verificaram melhores escores nos domínios físico, psicológico e na percepção geral da QV.

Os aspectos físicos e psicológicos relacionados a QV podem ser percebidos de forma negativa por pessoas com DV, ao levar-se em consideração a falta de oportunidade para a prática esportiva e de lazer, a superproteçáo de pais e professores, além do preconceito $^{31}$ e dificuldade de adaptação na sociedade ${ }^{32}$. Nesse sentido, a prática de exercícios físicos torna-se muito importante, na medida em que os influencia positivamente como evidenciado no presente estudo.

Destaca-se, também, a importância que os exercícios físicos podem ter no relacionamento social entre os participantes. McDonnall ${ }^{33}$ destaca que adultos com DV apresentam melhores condições psicológicas para enfrentar os desafios diários e manter um estilo de vida mais saudável quando estão inseridos em grupos e em relacionamentos gerais. No estudo, o domínio das relaçôes sociais já apresentava uma média elevada no início do treinamento, podendo ser classificado como "muito bom". Ainda assim, é possível observar um aumento nos valores mínimos, indicando efeitos positivos do treinamento.

No estudo de Movahedi et al. ${ }^{34}$ foram evidenciados escores mais elevados de socialização entre os adolescentes com DV que eram atletas. Em estudos realizados com idosos chineses ${ }^{17,35}$ e idosos canadenses $^{18}$, a DV e a QV estavam diretamente ligadas com o convívio social em que, ter redes de amizade estavam associadas a uma melhor QV.

Ao que se refere a percepção geral da QV e a satisfação com a saúde, os participantes já apresentavam no início do estudo, igualmente, valores que indicavam uma boa percepção. Ainda assim, foi verificado o aumento nos valores mínimos após o treinamento. A percepção de saúde também é um importante componente para a definição de QV e, no caso de pessoas com DV pode ser influenciada pela aceitação ou não da deficiência ${ }^{36}$.

No seu estudo, Scherer ${ }^{37}$ não encontrou associaçóes entre o resquício visual, o sexo e a percepção da QV dos adultos com deficiência visual de Florianópolis, no entanto, observou que quanto maior o tempo que as pessoas tinham adquirido a DV, melhor era a sua percepção da QV. Apesar de o presente estudo não ter buscado verificar essas relações, percebe-se que apenas o sujeito "I" tinha adquirido a deficiência há dois anos e, os demais, há mais de cinco anos, fato este que pode justificar a boa percepção dos participantes.

Sabe-se que o ambiente que as pessoas vivem também influencia na percepção da QV. O domínio meio ambiente engloba as condiçóes ambientais do lugar onde moram e circulam, tais como saneamento básico, poluição sonora, violência, meio de transporte, etc. Em se tratando de pessoas com DV, estes fatores podem ser agravados pela falta de acessibilidade em ir até locais como farmácias, supermercados, faculdade, trabalho, etc., além da falta de ambientes adequados para realizar atividades de lazer e falta de segurança para locomoção. No presente estudo, não houve melhoras estatisticamente significativas neste domínio.

No estudo de SCHERER ${ }^{37}$ apesar de a maioria das pessoas com DV ter relatado percepçóes positivas acerca do saneamento básico, acessibilidade a farmácias, ponto de ônibus, supermercados, etc., também relataram ter dificuldades e alguns aspectos negativos com os mesmos. Estes fatores que podem dificultar a mobilidade destas pessoas e diminuir a percepçáo positiva acerca do ambiente, podem acarretar a perda de emprego, redução de atividades recreativas, perda de autossuficiência ${ }^{33}$, ou seja, estas barreiras influenciam diretamente na QV dessas pessoas $^{38}$ e consequentemente no TAF.

Considera-se como limitantes deste estudo o pequeno número de participantes, a ampla faixa etária, a ausência de um grupo controle não treinando em função do pequeno número de participantes e 
associação de outras deficiências junto à deficiência visual, e variadas características da deficiência, não sendo possível generalizar-se os resultados para a população em geral de adultos com DV. A indisponibilidade de horários dos participantes permitiu a realização de apenas duas sessóes semanais, não atendendo as recomendaçôes de 150 minutos de atividades semanais ${ }^{39}$ (ACSM, 2006). Além disso, a modificação da intensidade dos exercícios a partir da percepção subjetiva de esforço dos participantes pode não ter sido eficaz.

Até o momento, este parece ser o primeiro estudo que buscou avaliar o efeito de um programa de exercícios físicos combinados no TAF e QV de adultos com DV.

Conclui-se que o programa de exercícios combinados, com pesos e aeróbicos, promoveu melhoria no TAF e nos domínios físico e psicológico da QV dos adultos com DV. Neste sentido, é relevante que a Educação Física também se aproprie de programas de exercícios físicos com atividades combinadas, a fim de diversificar suas práticas e promover benefícios à saúde desta população. Desta forma, faz-se possível promover maior independência para a pessoa com DV, possibilitando a adesão a um estilo de vida mais ativo e uma melhor QV.

\section{Abstract}

\section{Combined exercise in adults with visual impairment}

The purpose of this study was to evaluate the effects of a combined physical exercise program on the physical activity time (PAT) and quality of life $(\mathrm{OOL})$ of adults with visual impairment $(\mathrm{VI})$. A group of nine visually impaired subjects, with a mean age of $44.9 \pm 11.3$ years were studied. A functional training program with resistance and aerobic exercises combined in a single session was performed twice a week during a 12-week period, in a Physical Activity Studio. The PAT was assessed using the IPAQ, short version and the QOL by WHOOOL - short bref in the Portuguese version. An anamnesis was also used to characterize the participants. The evaluations were made before the training period and afterwards. The Shapiro-Wilk test was used to verify the data distribution and the Wilcoxon test to evaluate the difference between the mean values obtained before and after training. Statistically significant improvements were found in PAT and physical and psychological domains of QOL. It was concluded that combined physical exercises improve $\mathrm{QOL}$ and PAT, enabling a more active lifestyle and independence in people with VI.

KeYwords: Aerobic Exercise; Functional Resistance Exercise; Time of Physical Activity; Quality of Life; People with Disabilities.

\section{Referências}

1. Instituto Brasileiro de Geografia e Estatística. Censo demográfico 2010: características gerais da população: resultados da amostra. Rio de Janeiro: IBGE; 2010.

2. Munster MA, Almeida JJG. Atividade física e deficiência visual. In: Goragatti MG, Costa RF. Atividade física adaptada: qualidade de vida para pessoas com necessidades especiais. 2a ed. Barueri: Manole; 2008. Cap. 2; p. 28-75.

3. World Health Organization. International statistical classification of diseases and related health problems [Internet]. 10th revision. Geneva: WHO; 2005 [citado em 2 mar 2018]. Disponível em: <https:/goo.gl/nKag5e>.

4. Almeida MB, Araújo CGS. Efeitos do treinamento aeróbico sobre a frequência cardíaca. Rev Bras Med Esporte. 2003;9(2):104-12.

5. Fagard RH, Cornelissen VA. Effect of exercise on blood pressure control in hypertensive patients. Eur J Cardiovasc Prev Rehabil. 2007;14(1):12-7.

6. Teixeira L. Atividade física adaptada e saúde. $1^{\mathrm{a}}$ ed. São Paulo: Phorte; 2008.

7. Nahas MV. Atividade física, saúde e qualidade de vida: conceitos e sugestôes para um estilo de vida ativo. $5^{\text {a }}$ ed. Londrina: Midiograf; 2010. 
8. Caspersen CJ, Powell KE, Christensen GM. Physical activity, exercise, and physical fitness: definitions and distinctions for health-related research. Public Health Rep. 1985;100(2):126-31.

9. Rimmer JH, Riley B, Wang E, Rauworth A, Jurkowski J. Physical activity participation among persons with disabilities: barriers and facilitators. Am J Prev Med. 2004;26(5):419-25.

10. Ackley EI, Caputo JL, Perry TL, Fuller DK, Morgan D. Physical activity, body composition, and perceived quality of life of adults with visual impairments. J Visual Impair Blin. 2009;103(1):17-29.

11. Bittencourt ZZLC, Hoehne EL. Qualidade de vida de deficientes visuais. Med Ribeirão Preto. 2006;39(2):260-4.

12. Araújo DSMS, Araújo CGS. Aptidáo física, saúde e qualidade de vida relacionada à saúde em adultos. Rev Bras Med Esporte. 2000;6(5):194-203.

13. Silva GP, Pereira VR, Deprá PP, Gorla JI. Tempo de reação e a eficiência do jogador de goalball na interceptação/defesa do lançamento/ataque. Motricidade. 2010;6(4):13-22.

14. Campos MO, Maciel MG, Rodrigues Neto JF. Atividade física insuficiente: fatores associados e qualidade de vida. Rev Bras Ativ Fis e Saúde. 2012;17(6):562-72.

15. Lima CRF, Gorgatti MG, Dutra MC. A influência do esporte na qualidade de vida das pessoas com deficiência visual. RAS. 2010;8(23):40-7.

16. Interdonato GC, Greguol M. Qualidade de vida e prática habitual de atividade física em adolescentes com deficiência. Rev Bras Crescimento Desenvolv Hum. 2011;21(2):282-95.

17. Wang C, Chan CL, Ho AH, Xiong Z. Social networks and health-related quality of life among Chinese older adults with vision impairment. J Aging Health. 2008;20(7):804-23.

18. Renaud J, Levasseur M, Gresset J, Overbury O, Wanet-Defalque MC, Dubois MF, et al. Health-related and subjective quality of life of older adults with visual impairment. Disabil Rehabil. 2010;32(11):899-907.

19. Mendonça GV, Pereira FD, Fernhall B. Effects of combined aerobic and resistance exercise training in adults with and without down syndrome. Arch Phys Med Rehabil. 2011;92(1):37-45.

20. Calders P, Elmahgoub S, Mettelinge TR, Vandenbroeck C, Dewandele I, Rombaut L, et al. Intellectual disability: a controlled trial effect of combined exercise training on physical and metabolic fitness in adults with intellectual disability: a controlled trial. Clin Rehabil. 2011;25:1097-108.

21. Nunciato AC, Pastrelo D, Leite RD, Prestes J, Medalha CC. Treinamento de força e treinamento funcional em adolescente lesado medular: relato de caso. ConScientiae Saúde. 2009;8(2):281-88.

22. Alves JGB, Montenegro FMU, Oliveira FAO, Alves RV. Prática de esportes durante a adolescência e atividade física de lazer na vida adulta. Rev Bras Med Esporte. 2005;11(5):291-4.

23. Piccoli JC, Oliveira GT, Ferrareze ME. A prática de atividade física na região do Vale dos Sinos no Estado do Rio Grande do Sul. R Bras Ci Mov. 2010;18(1):42-7.

24. Łabudzki J, Tasiemski T. Physical activity and life satisfaction in blind and visually impaired individuals. Human Movement. 2013;14(3):210-6.

25. Borg GA. Psychophysical bases of perceived exertion. Med Sci Sports Exerc. 1982;14(5):377-81.

26. Nogueira CR, Shibata J, Gagliardi JFL. Comparação do equilíbrio estático e dinâmico entre atletas com deficiência visual, praticantes de goalball e atletismo. R Bras Ci Mov. 2009;17(2):[17 p.].

27. Matsudo S, Araújo T, Matsudo V, Andrade D, Andrade E, Oliveira LC, et al. Questionário internacional de atividade física (IPAQ): estudo de validade e reprodutibilidade no Brasil. Rev Bras Ativ Fís Saúde. 2001;6(2):5-18.

28. Fleck MPA, Louzada S, Xavier M, Chachamovich E, Vieira G, Santos L, et al. Aplicação da versão em português do instrumento abreviado de avaliação da qualidade de vida "WHOQOL-bref”. Rev Saúde Pública. 2000;34(2):178-83.

29. Heller T, Hsieh K, Rimmer JH. Attitudinal and psychosocial outcomes of a fitness and health education program on adults with down syndrome. Am J Ment Retard. 2004;109(2):175-85.

30. Montarzino A, Roberston B, Aspinall P, Ambrecht A, Findlay C, Hine J, et al. The impact of mobility and public transport on the independence of visually impaired people. Visual Impairment Research. 2007;9(2-3):67-82.

31. Lieberman LJ, Stuart ME, Hand KE. Beliefs about physical activity among children who are visually impaired and their parents. J Vis Impair Blind. 2006;100(4):223-34.

32. Lucas MB, Leal DB, Tavares SS, Barros EA, Aranha ST. Condutas reabilitacionais em pacientes com baixa visão. Arq Bras Oftalmol. 2003;66(1):77-82.

33. McDonnall MC. The need for health promotion for adults who are visually impaired. J Visual Impair Blin. 2007;101(3):133-45.

34. Movahedi A, Mojtahedi H, Farazyani F. Differences in socialization between visually impaired student-athletes and nonathletes. Res Dev Disabil. 2011;32(1):58-62. 
35. Zhang XC, Wang A, Yin A. The impact of psychosocial adaptation status on quality of life for Chinese patients with visual impairments. J Clin Nurs. 2012;23(1-2):75-81.

36. Rimmerman A, Morgenstern H. Quality of life of visually impaired adults who are employment programs in Israel. J Vis Impair Blind. 2003;97(9):551-61.

37. Scherer RL. Qualidade de vida de adultos com deficiência visual da grande Florianópolis [dissertação]. Florianópolis: Universidade Federal de Santa Catarina; 2012.

38. Kirchner CE, Gerber EG, Smith BC. Community barriers to physical activity for people with visual or motor impairments. Am J Prev Med. 2008;34(4):349-52.

39. Americam College of Sports Medicine (ACSM). Manual do ACSM para avaliação da aptidão física relacionada à saúde. Taranto G, tradutor. Rio de Janeiro: Guanabara Koogan; 2006.

ENDEREÇO

Rafaella Righes Machado

Universidade Federal de Santa Maria Av. Roraima, 1.00o - Cidade Universitária 97105-900 - Santa Maria - RS - Brasil e-mail: rafaellarighes@hotmail.com
Recebido para publicação: 23/04/2015

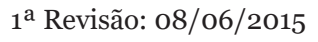

$2^{\text {a }}$ Revisão: 21/09/2015

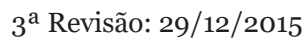

$4^{\mathrm{a}}$ Revisão: 01/02/2016

Aceito: 26/02/2016 